Review

\title{
Urinary $N$-Acetyl- $\beta$-D-glucosaminidase (uNAG) as an Indicative Biomarker of Early Diabetic Nephropathy in Patients with Diabetes Mellitus (T1DM, T2DM): A Systematic Review and Meta-Analysis
}

\author{
Arlinda R. Driza ${ }^{1}$ (D), Georgia V. Kapoula ${ }^{1,2}$ and Pantelis G. Bagos ${ }^{1, *(\mathbb{D})}$ \\ 1 Department of Computer Science and Biomedical Informatics, School of Science, University of Thessaly, \\ Papasiopoulou 2-4, 35100 Lamia, Greece; ae.driza@gmail.com (A.R.D.); gkapoula@hotmail.com (G.V.K.) \\ 2 Department of Biochemistry, General Hospital of Lamia, End of Papasiopoulou, 35100 Lamia, Greece \\ * Correspondence: pbagos@compgen.org; Tel.: +30-2231066914; Fax: +30-2231066915
}

check for

updates

Citation: Driza, A.R.; Kapoula, G.V.; Bagos, P.G. Urinary

$N$-Acetyl- $\beta$-D-glucosaminidase (uNAG) as an Indicative Biomarker of Early Diabetic Nephropathy in Patients with Diabetes Mellitus (T1DM, T2DM): A Systematic Review and Meta-Analysis. Diabetology 2021, 2, 272-285. https://doi.org/10.3390/ diabetology2040025

Academic Editor: Peter Clifton

Received: 25 October 2021

Accepted: 9 December 2021

Published: 15 December 2021

Publisher's Note: MDPI stays neutral with regard to jurisdictional claims in published maps and institutional affiliations.

Copyright: (c) 2021 by the authors. Licensee MDPI, Basel, Switzerland. This article is an open access article distributed under the terms and conditions of the Creative Commons Attribution (CC BY) license (https:/ / creativecommons.org/licenses/by/ $4.0 /)$.

\begin{abstract}
Diabetic nephropathy (DN) is the main cause of chronic kidney disease in patients with type 1 (T1DM) and type 2 diabetes mellitus (T2DM). Renal tubular lysosomal enzyme activities like $N$-acetyl- $\beta$-D-glucosaminidase (NAG) have been shown to increase in patients developing DN. The aim of this systematic review and meta-analysis is to evaluate the diagnostic accuracy of NAG, as a preventional biomarker in the early stages of DN in patients with diabetes mellitus. Two impartial reviewers conducted a complete PubMed search until July 2021. A $2 \times 2$ contingency table was created for each trial and sensitivity and specificity were estimated using a bivariate random effects model. To pool data and estimate the area under the curve (AUC), the hierarchical summary ROC (hsROC) approach was utilized. Deek's test was used to estimate publication bias. The meta-analysis included 21 studies that evaluated 2783 patients with T1DM and T2DM, as well as 673 healthy individuals. The AUC of urinary NAG (uNAG) ranged from 0.69 (95\% CI: $0.65-0.73$ ) to 0.89 (95\% CI: 0.86-0.92). According to the results, NAG in urine can be considered as a potential and effective biomarker for predicting DN in diabetic patients (T1DM, T2DM).
\end{abstract}

Keywords: $N$-acetyl- $\beta$-D-glucosaminidase (NAG); diabetic nephropathy; chronic kidney disease (CKD); meta-analysis; systematic review

\section{Introduction}

Diabetic nephropathy (DN), is a metabolic disease and one of the most frequent microvascular complications of type 1 (T1DM) and type 2 (T2DM) diabetes mellitus [1] The prolonged exposure of the body to high blood glucose levels (hyperglycemia) due to diabetes, affects proper functioning of the kidneys by damaging specific units responsible for removing waste products from the body and filtering essential substances to pass into the bloodstream [1]. DN is independently associated with cardiovascular risk in diabetic patients, especially in patients with T2DM [2]. Therefore, early detection and treatment is of major importance, as it can prevent critical complications of the disorder. DN is the leading cause of chronic kidney disease (CKD) and its diagnosis is based on the current level of albuminuria leading in three stages [3]: DN normoalbuminuria, microalbuminuria, and macroalbuminuria. More specifically, the confirmation of the disease is based on the persistent albuminuria in early morning urine samples, due to glomerular hyperfiltration [4,5]. Healthy individuals excrete small amount of albumin on a daily basis which does not exceed $30 \mathrm{mg} / \mathrm{g}$. Albumin-to-creatinine ratio (ACR) is the gold standard method to detect elevated protein excretion in urine samples of diabetic patients. The onset stage of DN is defined by moderately increased albuminuria, known as microalbuminuria and it is diagnosed by the detection of a significant amount of albumin in the urine, which ranges from 30-300 mg/24 h [5]. The progression of the disorder refers to a gradual decline in 
GFR and is characterized by severely abnormal increased levels of albuminuria, known as macroalbuminuria (proteinuria). It is increasingly appreciated that both glomerular and tubular interstitial damage have an essential role in the pathophysiology and development of DN [6].

However, according to recent studies, in 30\% of diabetic population diagnosed with microalbuminuria, the course of the disease has shifted to a new clinical picture, defined by normal albuminuria and GFR after 10 years of follow up [7-9]. Even though microalbuminuria measurement is the gold standard method to predict and monitor the progression of $\mathrm{DN}$, significant efforts have been made to investigate and validate alternative biomarkers for the diagnosis of DN, allowing the early identification of diabetic renal lesions [10]. Multiple biomarkers have been reported and classified due to their ability of detecting specific disorders [11]. Studies have shown promising preliminary results, suggesting that increased levels of the potential biomarkers are associated with the presence of renal damage in patients with T1DM and T2DM. According to studies, some of the effective biomarkers of glomerular injury are: adiponectin [12,13], transferrin [14], and ceruloplasmin [15]. Studies have also shown potential biomarkers which reflect tubular injuries, these include kidney injury molecule-1 KIM-1, a1- and b2-microglobulin [16-18], liver-type fatty acid binding protein L-FABP [17], and neutrophil gelatinase-associated lipocalin (NGAL). Recent meta-analysis suggests that NGAL is a potential valuable biomarker for early prediction of DN in diabetic patients [19].

In addition, $N$-acetyl- $\beta$-D-glucosaminidase (NAG) is a lysosomal enzyme found in proximal renal tubular cells and its significant concentrations during tubulointerstitial damage, are related with renal dysfunction [20]. In previous studies, increased urinary levels of NAG concentrations were present in diabetic patients diagnosed with normoalbuminuria rather than the control group. In addition, urinary NAG (uNAG) has been shown to increase progressively along with the DN stages, indicating that it might be an early predictive biomarker for DN [21,22]. Thus, the aim of this study is to conduct a systematic review and meta-analysis to evaluate the diagnostic accuracy of uNAG, as a preventional biomarker in the early stages of DN in patients with diabetic mellitus.

\section{Materials and Methods}

\subsection{Search Strategy and Study Selection}

This systematic review and meta-analysis was conducted according to the standard PRISMA (Preferred Reporting Item for Systematic Reviews and Meta-analyses) guidelines [23]. A literature search was performed on the PubMed database by two independent reviewers until the end of July 2021, using a clearly formulated query of terms and keywords ("urinary biomarker" OR "N-Acetyl-beta-D-Glucosamine" OR "GLcNAc" OR "N-AcetylGlucosamine" OR "urinary NAG" OR "serum NAG" OR "urinary lysosomal enzyme") AND ("diabetes" OR "diabetic nephropathy" OR “diabetic kidney disease"). To eliminate local literature bias, the study search was comprehensive and did not include language limitations. In addition, further search was conducted in other electronic engines, such as Google Scholar, the duplicates records were removed. The study selection was based on specific predefined criteria and each reason for inclusion or exclusion was recorded. All of the studies were included after reviewing the abstracts and full text of each article.

\subsection{Inclusion and Exclusion Criteria}

Studies chosen for meta-analysis were based on specific inclusion criteria. The rationale for the criteria of the study selection was predefined and clearly stated. The meta-analysis included studies, in which uNAG was determined in healthy individuals and in patients with diabetes mellitus. Diabetic patients were divided in the three following categories: patients with normoalbuminuria (UACR $<30 \mathrm{mg} / \mathrm{g}$ ), patients with microalbuminuria (UACR = 30-300 mg/g), and patients with macroalbuminuria (UACR > $300 \mathrm{mg} / \mathrm{g}$ ). Studies eligible for the meta-analysis also included the degree of DN determined by the 
estimation of the UACR using a 24-h urine sample, or a random morning urine sample, according to the American Diabetes Association [23].

\subsection{Data Extraction}

NAG concentration in urine samples and uNAG concentration normalized to the urinary creatinine (uNAG/Cr), were extracted from each study. Furthermore, data synthesis included the extraction of the first author's name, study location, year of publication, age and sex of the participant groups, type of diabetes and clinical characteristics of each study group. Finally, a $2 \times 2$ contingency table was constructed using the absolute data of true positive (TP), false positive (FP), true negative (TN), and false negative (FN) for comparing and combining the effects of different research.

\subsection{Quality Assessment of Safety Studies}

The study selection was performed guided by the mentioned criteria, followed by an in-depth quality assessment, using the Quality Assessment of Diagnostic Accuracy Studies 2 (QUADAS-2) [24]. The QUADAS tool consists of 14 questions of four different key domains. The risk of bias and the applicability of the studies were examined for each domain. The following judgements were used to complete the signaling questions: low, high, or unclear risk. For the quality analysis, the Review Manager Software (RevMan 5.4) was used.

\subsection{Meta-Analysis}

A random effects meta-analysis was performed in order to synthesize quantitative information from related studies. The approach method of the bivariate meta-analysis involves estimating sensitivity (logit-Se) and specificity (logit-Sp) by van Houwelingen $[25,26]$.

According to the available raw data, the following statistical calculations were used for estimating the mean and $S D$. Therefore, in the studies where the $95 \%$ confidence interval $(C I)$ was specified, $S E$ was determined according to the following equation recommended by Cochrane Handbook [22]:

$$
S E=(\text { upperlimit }- \text { lowerlimit }) / 3.92
$$

In the studies where median $(M)$ and inter-quartile range (IQR) were provided, for calculating mean and $S D$ we followed the Cochrane Handbook [27]. The median was utilized as a mean estimator, while the $S D$ was determined as follows:

$$
S D=\frac{I Q R}{1.35}
$$

In the studies where median $(M)$ and range were provided, we used the principles specified by Hozo and co-workers [28]. The following equation was used for sample sizes $n<25$ :

$$
\bar{x}=\frac{\min +2 M+\max }{4}
$$

In the studies where the sample size was $n>25$, median was chosen as the appropriate value over the mean value. $S D$ for sample sizes $n<15$ was calculated by the following equation:

$$
S D^{2}=\frac{1}{12}\left(\frac{(\min +2 M+\max )^{2}}{4}+(\max -\min )^{2}\right)
$$

while, for $n>25$ was calculated by the equation:

$$
S D=\frac{R}{4}
$$

The three following groups of diabetic patients were considered in the meta-analysis: patients with normoalbuminuria, patients with microalbuminuria, and patients with 
normo/microalbuminuria. Normoalbuminuria was defined using as status variables healthy individuals (controls) vs. normoalbuminuric patient group whereas for the prediction of microalbuminuria, normoalbuminuric vs. microalbuminuric patients, patient groups were used as status variables.

In order to assess the diagnostic performance of $\mathrm{uNAG}$ and $\mathrm{uNAG/Cr}$ in early diagnosis of DN, the hierarchical summary ROC curve (hsROC) was constructed using sensitivity, specificity, and parameters of the bivariate normal distribution. For each study, the absolute number of TPs, FPs, FNs, and TNs were computed by altering the threshold values (log cutoff) calculated by the raw extracted data from the articles, such as the mean and standard deviation (SD) of $\mathrm{uNAG}$ and $\mathrm{uNAG} / \mathrm{Cr}$, assuming a normal distribution. The Youden index at its maximum value, represents the ideal discrimination limit, which is calculated as $\mathrm{Y}=$ sensitivity + specificity -1 [29].

The interpretation of the curve was based on the following principals proposed by Swets [30]: Low $(0.5 \geq$ AUC $\leq 0.7)$, moderate $(0.7 \geq$ AUC $\leq 0.9)$, and high (0.9 $\geq$ AUC $\leq 1.0)$ accuracy. The between-study heterogeneity was estimated by using the Cochran Q-test and $\mathrm{I}^{2}$ statistic and was presented as a forest plot [31]. For the publication bias were used calculation methods according to Deek's et al. [32]. The current study's data synthesis and statistical analysis were carried out using Stata software v.13 (College Station, TX, USA: StataCorp LLC).

\section{Results}

\subsection{Included Studies and Trial Characteristics}

Literature search from the databases yielded 353 citations, of which 3 were duplicates and discarded, resulting in 350 unique citations. Following the first review of the titles and abstracts, 283 articles were excluded for not meeting the inclusion criteria. Further, following a detailed examination of the 69 full-text articles, 48 articles were removed due to misclassification of DN or the unclear statement of the methods used in the study. Consequently, a total of 21 studies were found eligible and were included in the metaanalysis. The PRISMA flow of the review process is shown in Figure 1. These studies consisted of 2783 patients and 673 healthy in total. Table 1 represents the population's characteristics extracted from each study. Precisely, studies included 1196 patients with T1DM, 1587 patients with T2DM, and 673 healthy individuals. The group of patients with T1DM involved 644 patients $(53.8 \%)$ with normoalbuminuria, 477 patients with microalbuminuria (39.8\%), and 75 patients $(6.3 \%)$ with macroalbuminuria. The group of patients with T2DM involved 760 patients (47.8\%) with normoalbuminuria, 663 patients (41.7\%) with microalbuminuria, and 164 patients $(10.3 \%)$ with macroalbuminuria. Patients with macroalbuminuria were not included in the meta-analysis due to the insufficient number of studies. Furthermore, $50.5 \%$ of the patient group, were male with a mean age of 53.4 years old. In the healthy group $50.1 \%$ were male with a mean age of 51.3 years old. The clinical features collected from the studies are expressed by the weighted average and the concentrated standard deviation and are presented in supplementary Table S1. 
Table 1. Detailed characteristics of the included studies in the meta-analysis for controls and diabetic patients.

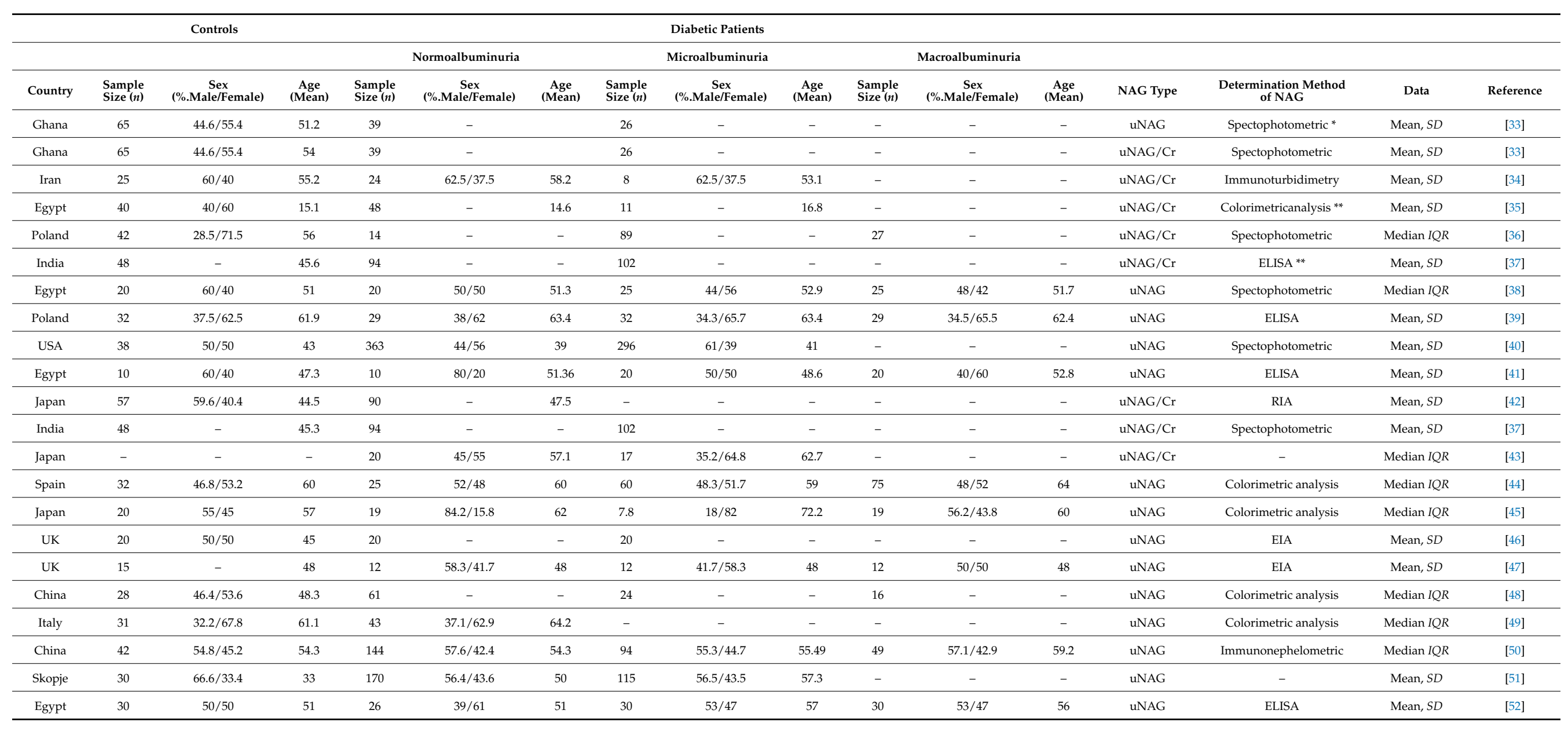

* NAG absorbance for spectophotometric analysis: OD = 400-405 nm ** NAG absorbance for ELISA/colorimetric analysis: OD = 400 nm. 


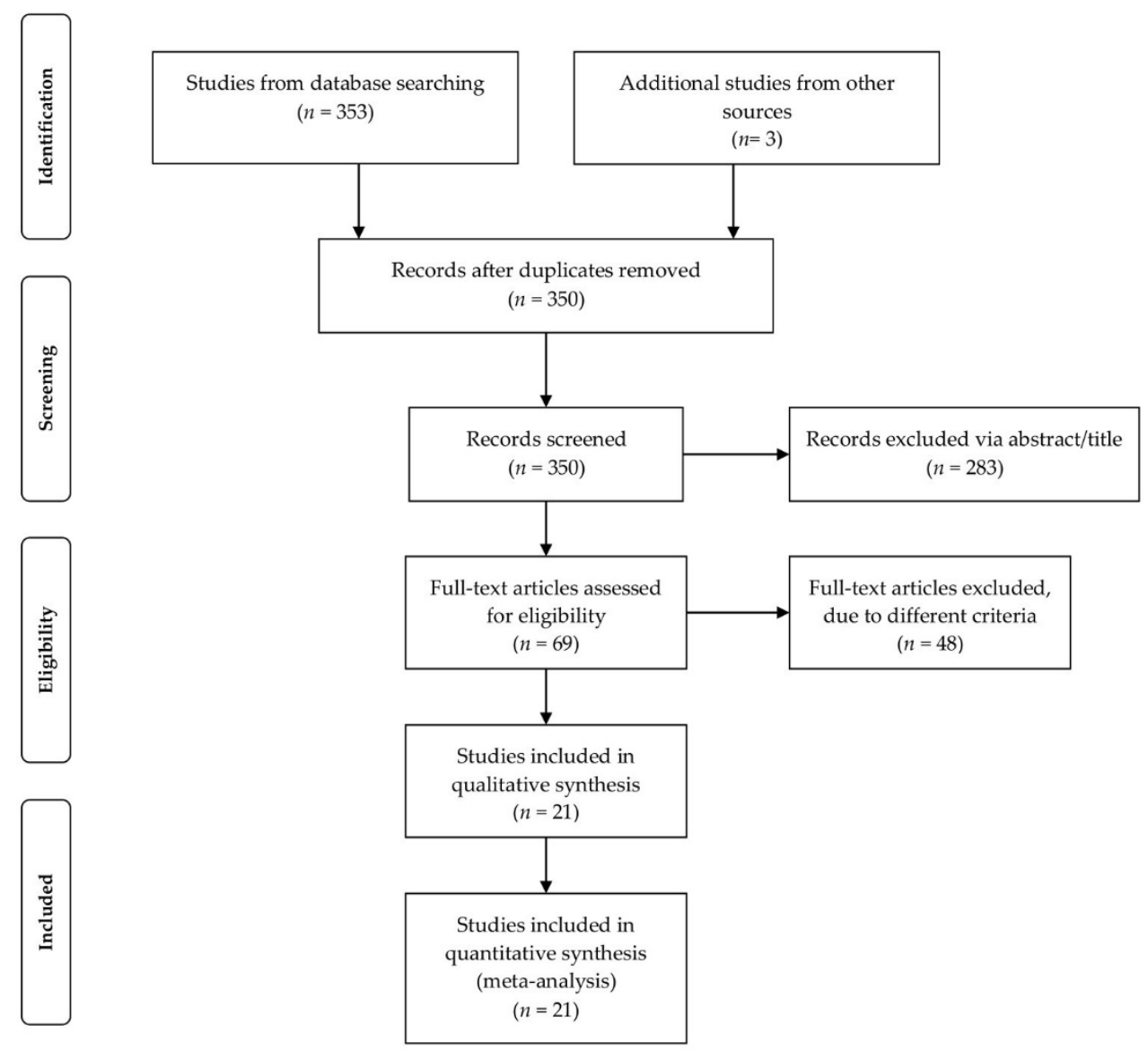

Figure 1. PRISMA flow diagram for literature search and study selection.

\subsection{Quality Assessment of the Included Studies}

The outcome of the comprehensive quality assessment of the 21 included studies are shown in the Figure 2. The unclear risk of bias in some studies on patient selection was present due to lack of information about the characteristics of the patient group with microalbuminuria and macroalbuminuria, such as the total number of patients, age, and gender. There was a concern regarding the applicability of the index test due to the different processing of the uNAG sample.

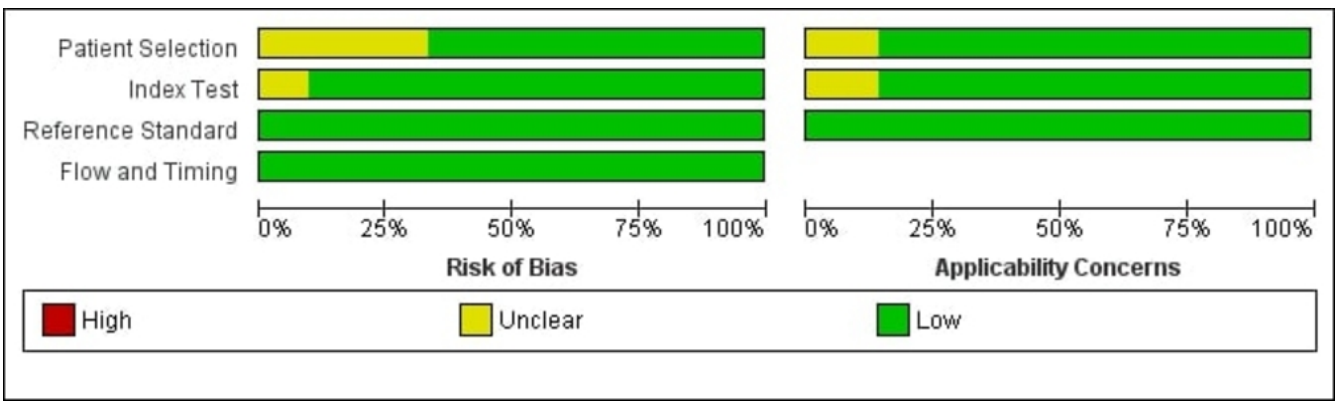

Figure 2. The Quality Assessment of Diagnostic Accuracy Studies-QUADAS. The figure represents the risk of bias and the applicability concern for the included studies. Each risk of bias is illustrated as percentage $(\%)$.

\subsection{Diagnostic Accuracy and Summary ROC Curve}

Overall, pooled sensitivity and specificity in diabetic patients, ranged from $0.65(95 \%$ CI: $0.38-0.85)$ to 0.84 (95\% CI: $0.77-0.89)$ and 0.65 (95\% CI: $0.41-0.83)$ to 0.88 (95\% CI: $0.67-0.97$ ) respectively. An hsROC curve was created for each category (uNAG, uNAG/Cr) 
and the AUC was calculated, along with the $95 \%$ CI. The diagnostic accuracy of uNAG estimated by AUC for predicting DN in diabetic patients, for all groups ranged from 0.69 (95\% CI: 0.65-0.73) to 0.89 (95\% CI: 0.86-0.92) (Figure 3 and supplementary Figures S1-S2). These results show moderate to excellent diagnostic accuracy of uNAG and uNAG/Cr. In addition, the best predictive performance was shown by uNAG and uNAG/Cr to discriminate between diabetic patients with normo-microalbuminuria and the healthy group with an AUC $=0.89$ (95\% CI: 0.86-0.92) (Figure 3). In the meta-analysis were used the following values, which are presented in Table 2: TP, FN, FP, and TN, paired sensitivity and specificity, along with the corresponding $95 \% \mathrm{CI}$ and the cutoff values for each individual study. Table 3 provides the diagnostic and prognostic values for uNAG and $\mathrm{uNAG} / \mathrm{Cr}$.

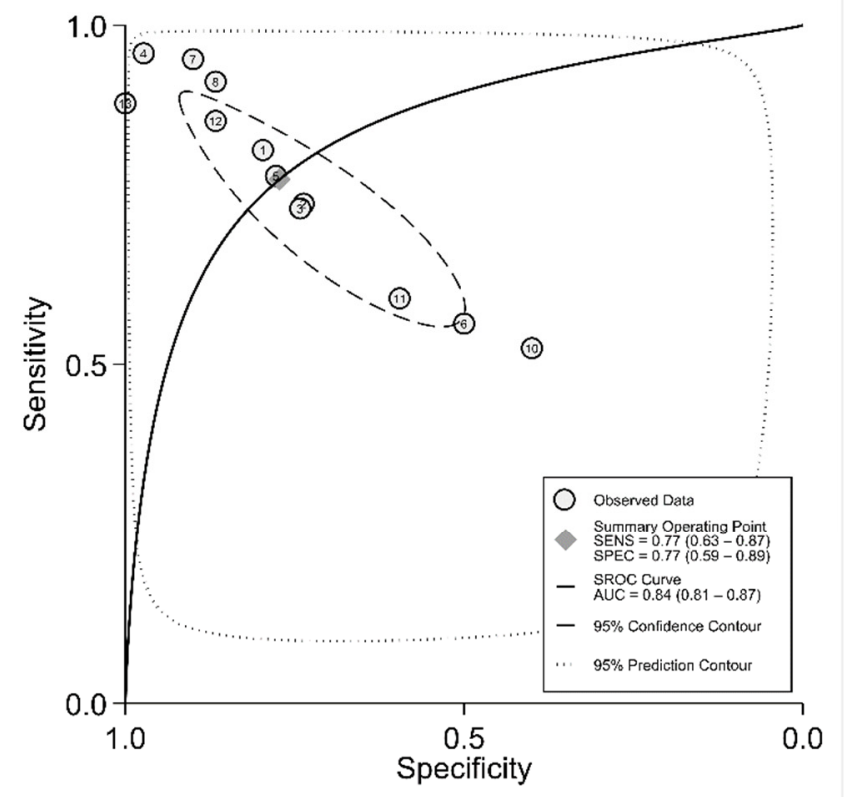

(a)

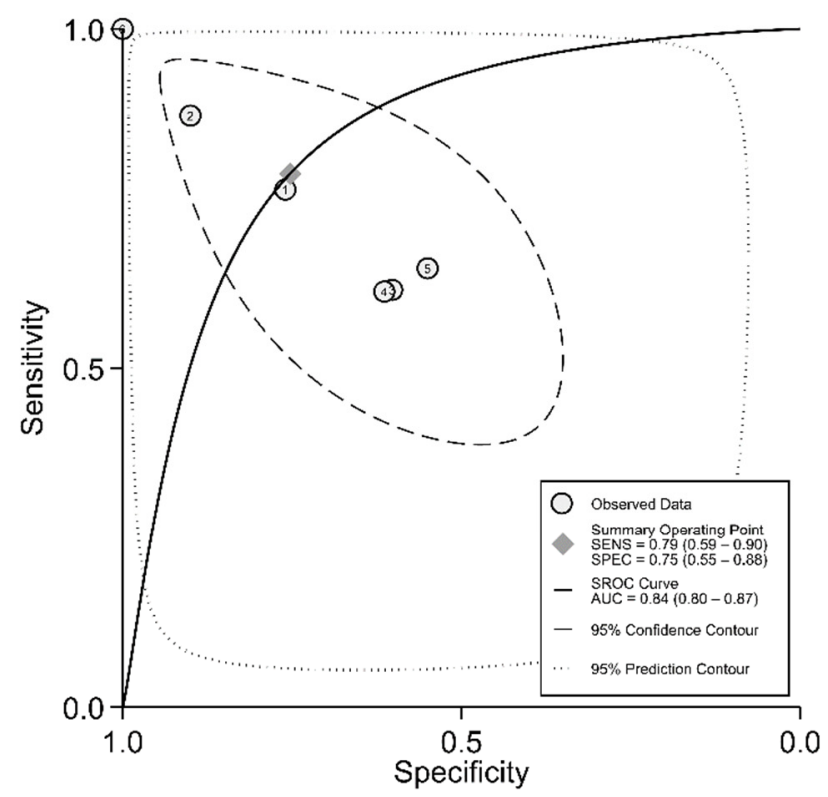

(b)

Figure 3. The hierarchical summary Receiver Operating Characteristic (hsROC) curve of uNAG (a) and uNAG/Cr (b) to differentiate controls (healthy individuals) from normoalbuminuric diabetic patients. 
Table 2. Contingency table for uNGAL and uNGAL/Cr in diabetic patients, along with paired sensitivity and specificity of individual studies.

\begin{tabular}{|c|c|c|c|c|c|c|c|c|c|c|c|}
\hline \multicolumn{12}{|c|}{ uNAG: Controls vs. Patients with Normoalbuminuria } \\
\hline PubMed ID & Author Name & Country & Year & Type of Diabetes & Cut-Off & $\mathrm{TP} *$ & $\mathrm{FN}^{*}$ & $\mathrm{TN}^{*}$ & $\mathbf{F P} *$ & Sensitivity $(95 \% . \mathrm{CI})$ & Specificity $(95 \% \mathrm{CI})$ \\
\hline 27594733 & Anane H.A. & Ghana & 2016 & 2 & 11.15 & 31 & 7 & 51 & 13 & $0.80(0.63-0.90)$ & $0.79(0.68-0.88)$ \\
\hline 23966807 & Heba S. Assal & Egypt & 2013 & 2 & 8.25 & 14 & 5 & 14 & 5 & $0.72(0.48-0.90)$ & $0.72(0.48-0.90)$ \\
\hline 25519006 & Zurawska Plaksej E. & Poland & 2014 & 2 & 156.5 & 27 & 10 & 23 & 8 & $0.73(0.56-0.86)$ & $0.71(0.53-0.86)$ \\
\hline 20980978 & Vaidya S. V. & USA & 2011 & 1 & 1.15 & 347 & 15 & 36 & 1 & $0.95(0.92-0.97)$ & $0.96(0.86-0.99)$ \\
\hline 25717442 & Gehan S. & Egypt & 2015 & 2 & 1 & 7 & 2 & 7 & 2 & $0.70(0.34-0.93)$ & $0.70(0.34-0.93)$ \\
\hline 16935891 & Navarro J.F. & Spain & 2006 & 1 & 1 & 14 & 11 & 16 & 16 & $0.56(0.34-0.76)$ & $0.50(0.32-0.68)$ \\
\hline 17910281 & Kalansoopiya A. & UK & 2007 & 2 & 1 & 19 & 1 & 18 & 2 & $0.95(0.75-0.99)$ & $0.90(0.68-0.98)$ \\
\hline 21779943 & Fu W. & China & 2011 & 2 & 1 & 11 & 49 & 3 & 24 & $0.18(0.09-0.30)$ & $0.11(0.02-0.29)$ \\
\hline 26904288 & Muro P.D. & Italy & 2015 & 2 & 1 & 22 & 20 & 16 & 24 & $0.52(0.36-0.68)$ & $0.40(0.24-0.56)$ \\
\hline 31218128 & Zhang D. & China & 2019 & 2 & 1 & 86 & 58 & 25 & 17 & $0.59(0.51-0.67)$ & $0.40(0.25-0.56)$ \\
\hline- & Nikolov G. & Skopje & 2013 & 2 & 1 & 146 & 24 & 26 & 4 & $0.85(0.79-0.90)$ & $0.86(0.69-0.96)$ \\
\hline 32601635 & Shrouq F.A.H. & Egypt & 2020 & 2 & 1 & 23 & 3 & 30 & 0 & $0.88(0.69-0.97)$ & $1.00(0.88-1.00)$ \\
\hline \multicolumn{12}{|c|}{ uNAG/Cr: Controls vs. Patients with Normoalbuminuria } \\
\hline PubMed ID & Author Name & Country & Year & Type of Diabetes & Cut-Off & TP & FN & TN & FP & Sensitivity $(95 \% \mathrm{CI})$ & Specificity $(95 \% \mathrm{CI})$ \\
\hline 27594733 & Anane H.A. & Ghana & 2016 & 2 & 9.2 & 22 & 17 & 38 & 27 & $0.56(0.39-0.72)$ & $0.58(0.45-0.70)$ \\
\hline 15016173 & Salem M. A. K. & Egypt & 2002 & 1 & 4.6 & 38 & 10 & 31 & 9 & $0.79(0.65-0.89)$ & $0.77(0.61-0.89)$ \\
\hline 2881186 & Shimojo N. & Japan & 1987 & 1 & 2.3 & 99 & 1 & 56 & 1 & $1.00(0.95-1.00)$ & $1.00(0.95-1.00)$ \\
\hline 23105632 & Ambade V. & India & 2003 & 1 & 6.2 & 65 & 29 & 33 & 15 & $0.68(0.53-0.81)$ & $0.69(0.58-0.78)$ \\
\hline 16641878 & Piwowar A. & Poland & 2006 & 2 & 0.3 & 9 & 17 & 27 & 35 & $0.34(0.17-0.55)$ & $0.43(0.31-0.56)$ \\
\hline 18022929 & Karakani A. M. & Iran & 2007 & 1 & 3.6 & 23 & 1 & 24 & 1 & $1.00(0.85-1.00)$ & $1.00(0.85-1.00)$ \\
\hline \multicolumn{12}{|c|}{ uNAG: Patients with Normoalbuminuria vs. Patients with Microalbuminuria } \\
\hline PubMed ID & Author Name & Country & Year & Type of Diabetes & Cut-Off & $\mathbf{T P}$ & FN & TN & FP & Sensitivity $(95 \% \mathrm{CI})$ & Specificity $(95 \% \mathrm{CI})$ \\
\hline 27594733 & Anane H.A. & Ghana & 2016 & 2 & 12.9 & 2 & 1 & 1 & 1 & $0.53(0.37-0.69)$ & $0.52(0.33-0.73)$ \\
\hline 23966807 & Heba S. Assal & Egypt & 2013 & 2 & 13.8 & 1 & 3 & 2 & 4 & $0.76(0.50-0.91)$ & $0.91(0.73-0.99)$ \\
\hline 25519006 & Zurawska Plaksej E. & Poland & 2014 & 2 & 193.5 & 2 & 1 & 2 & 1 & $0.54(0.38-0.71)$ & $0.48(0.29-0.65)$ \\
\hline 16966829 & Fujita $\mathrm{H}$. & Japan & 2006 & 2 & 20 & 18 & 0 & 19 & 0 & $1.00(0.81-1.00)$ & $1.00(0.82-1.00)$ \\
\hline 25717442 & Gehan S. & Egypt & 2015 & 2 & 1.2 & 6 & 3 & 12 & 7 & $0.62(0.26-0.87)$ & $0.60(0.36-0.80)$ \\
\hline 16935891 & Navarro J.F. & Spain & 2006 & 1 & 4 & 34 & 26 & 14 & 11 & $0.56(0.43-0.69)$ & $0.56(0.34-0.75)$ \\
\hline 21779943 & Fu W. & China & 2011 & 2 & 12.7 & 16 & 8 & 41 & 20 & $0.66(0.44-0.84)$ & $0.67(0.54-0.78)$ \\
\hline 20980978 & Vaidya S.V. & USA & 2011 & 1 & 2.5 & 2 & 6 & 2 & 4 & $0.82(0.78-0.86)$ & $0.84(0.79-0.88)$ \\
\hline
\end{tabular}


Table 2. Cont

\begin{tabular}{|c|c|c|c|c|c|c|c|c|c|c|c|}
\hline \multirow[b]{2}{*}{ PubMed ID } & \multirow[b]{2}{*}{ Author Name } & \multirow[b]{2}{*}{ Country } & \multicolumn{9}{|c|}{ uNAG/Cr: Patients with Normoalbuminuria vs. Patients with Microalbuminuria } \\
\hline & & & Year & Type of Diabetes & Cut-Off & TP & FN & TN & FP & Sensitivity $(95 \% \mathrm{CI})$ & Specificity $(95 \% \mathrm{CI})$ \\
\hline 27594733 & Anane H.A. & Ghana & 2016 & 2 & 15 & 29 & 9 & 19 & 6 & $0.76(0.60-0.88)$ & $0.75(0.56-0.91)$ \\
\hline 15016173 & Salem M. A. K. & Egypt & 2002 & 1 & 9.8 & 41 & 6 & 9 & 1 & $0.85(0.72-0.93)$ & $0.87(0.58-0.99)$ \\
\hline 23105632 & Ambade V. & India & 2003 & 1 & 9.6 & 57 & 36 & 62 & 39 & $0.61(0.51-0.71)$ & $0.60(0.50-0.70)$ \\
\hline 16373913 & Narita T. & Japan & 2005 & 2 & 3 & 11 & 6 & 11 & 9 & $0.67(0.38-0.85)$ & $0.55(0.31-0.76)$ \\
\hline 18022929 & Karakani A. M. & Iran & 2007 & 1 & 6.2 & 23 & 0 & 7 & 0 & $1.00(0.85-1.00)$ & $1.00(0.85-1.00)$ \\
\hline \multicolumn{12}{|c|}{ uNAG: Controls vs. Patients with Normo-Microalbuminuria } \\
\hline 23966807 & Heba S. Assal & Egypt & 2013 & 2 & 10 & 38 & 7 & 19 & 1 & $0.84(0.70-0.93)$ & $0.93(0.75-0.99)$ \\
\hline 25519006 & Zurawska Plaksej E. & Poland & 2014 & 2 & 160 & 53 & 17 & 23 & 9 & $0.75(0.63-0.85)$ & $0.72(0.53-0.86)$ \\
\hline 20980978 & Vaidya S. V. & USA & 2011 & 1 & 1.3 & 597 & 62 & 38 & 0 & $0.90(0.88-0.92)$ & $1.00(0.90-1.00)$ \\
\hline 25717442 & Gehan S. & Egypt & 2015 & 2 & 1 & 21 & 9 & 8 & 2 & $0.70(0.50-0.85)$ & $0.79(0.44-0.97)$ \\
\hline \multicolumn{12}{|c|}{ uNAG /Cr: Controls vs. Patients with Normo-Microalbuminuria } \\
\hline PubMed ID & Author Name & Country & Year & Type of Diabetes & Cut-Off & TP & FN & TN & FP & Sensitivity $(95 \% \mathrm{CI})$ & Specificity $(95 \% \mathrm{CI})$ \\
\hline 15016173 & Salem M. A. K. & Egypt & 2002 & 1 & 5.2 & 53 & 6 & 33 & 7 & $0.89(0.79-0.96)$ & $0.82(0.67-0.92)$ \\
\hline 18022929 & Karakani A. M. & Iran & 2007 & 1 & 4 & 32 & 0 & 25 & 0 & $1.00(0.89-1.00)$ & $1.00(0.89-1.00)$ \\
\hline 23105632 & Ambade V. & India & 2003 & 1 & 6.5 & 142 & 54 & 34 & 14 & $0.72(0.65-0.78)$ & $0.70(0.55-0.83)$ \\
\hline 27594733 & Anane H.A. & Ghana & 2016 & 2 & 11 & 45 & 20 & 44 & 21 & $0.69(0.56-0.80)$ & $0.68(0.54-0.78)$ \\
\hline
\end{tabular}

* TP: True Positive, * FN: False Negative, * TN: True Negative, * FP: False Positive. 
Table 3. Pooled diagnostic and prognostic accuracy of uNAG in T1DM and T2DM patients.

\begin{tabular}{|c|c|c|c|c|c|c|c|c|c|}
\hline Number of Studies & Sensitivity $(95 \% \mathrm{CI})$ & $I^{2}(\%)$ & Specificity $(95 \% \mathrm{CI})$ & $I^{2}(\%)$ & PLR $(95 \% \mathrm{CI})$ & NLR $(95 \%$ CI $)$ & DOR $(95 \% \mathrm{CI})$ & AUC (95\% CI) & $p$-Value \\
\hline \multicolumn{10}{|c|}{ uNAG: Controls vs. patients with normoalbuminuria } \\
\hline 13 & $0.77(0.63-0.87)$ & $64.65(37.83-91.46)$ & $0.77(0.59-0.89)$ & $58.22(25.48-90.96)$ & $3.4(1.5-7.6)$ & $0.29(0.14-0.06)$ & $12(3-52)$ & $0.84(0.81-0.87)$ & 0.89 \\
\hline \multicolumn{10}{|c|}{ uNAG/Cr: Controls vs. patients with normoalbuminuria } \\
\hline 7 & $0.82(0.56-0.94)$ & $93.22(89.64-96.80)$ & $0.79(0.57-0.92)$ & $93.95(90.87-97.04)$ & $3.9(1.4-11.1)$ & $0.23(0.07-0.79)$ & $17(2-159)$ & $0.87(0.84-0.90)$ & 0.63 \\
\hline \multicolumn{10}{|c|}{ uNAG: Patients with normoalbuminuria vs. patients with microalbuminuria } \\
\hline 8 & $0.65(0.38-0.85)$ & $64.65(37.83-91.46)$ & $0.65(0.41-0.83)$ & $58.22(25.48-90.96)$ & $1.8(0.7-4.8)$ & $0.54(0.20-1.49)$ & $3(0-24)$ & $0.69(0.65-0.73)$ & 0.66 \\
\hline \multicolumn{10}{|c|}{ uNAG/Cr: Patients with normoalbuminuria vs. patients with microalbuminuria } \\
\hline 6 & $0.79(0.59-0.90)$ & $82.49(69.37-95.61)$ & $0.75(0.55-0.88)$ & $85.76(75.66-95.87)$ & $3.2(1.4-7.4)$ & $0.28(0.11-0.70)$ & $11(2-61)$ & $0.84(0.80-0.87)$ & 0.13 \\
\hline \multicolumn{10}{|c|}{ uNAG: Controls vs. patients with normo-microalbuminuria } \\
\hline 4 & $0.83(0.73-0.89)$ & $87.99(78.95-97.04)$ & $0.92(0.66-0.99)$ & $74.65(51.70-97.59)$ & $10.8(1.9-61.9)$ & $0.19(0.11-0.33)$ & $58(6-540)$ & $0.90(0.88-0.93)$ & 0.49 \\
\hline \multicolumn{10}{|c|}{ uNAG/Cr: Controls vs. patients with normo-microalbuminuria } \\
\hline 5 & $0.84(0.56-0.95)$ & $96.43(94.53-98.32)$ & $0.81(0.48-0.95)$ & 93.13 (88.69-97.56] & $4.4(1-19]$ & $0.20(0.05-0.85)$ & $22(1-388)$ & $0.89(0.86-0.92)$ & 0.08 \\
\hline
\end{tabular}




\subsection{Subgroup Analysis and Publication Bias}

The forest plot graphical presentation had shown notable heterogeneity in sensitivity and specificity in all sets examined. The degree of heterogeneity in sensitivity and specificity for all groups ranged from $64.65 \%$ to $96.43 \%$ and from $58.22 \%$ to $93.95 \%$, correspondingly. The between study heterogeneity for the prediction of normoalbuminuria and microalbuminuria in diabetic patients is presented in supplementary Figures S3-S5. The Deek's funnel plot estimated the evaluation of publication bias, in which $p$-value for all the groups, ranged from 0.08 to 0.89 and is showed in supplementary Figures S6-S8. Potential bias was present in the studies with data involving uNAG, specifically for distinguishing controls and normoalbuminuric or microalbuminuric diabetic patients.

\section{Discussion}

Diabetic nephropathy is considered to be the main cause of end-stage renal disease and a critical complication in patients with T1DM and T2DM. Therefore, the prediction of the disease at an early stage of its development is considered of the highest value. The diagnosis of $\mathrm{DN}$ is based on microalbuminuria estimation [5]. However, according to recent studies, diabetic patients diagnosed with microalbuminuria, have shifted back to normoalbuminuria and high GFR [7-9]. In addition, pathogenesis of DN evolves an interaction between metabolic and hemodynamic factors which cause glomerular and tubular interstitial injury. Therefore, researchers have been questioning and reevaluating the diagnostic value of the gold standard method, proposing an alternative approach, using potential tubular biomarkers such as NAG, for the early prediction of DN or other glomerular and markers of oxidative stress or inflammation. The attempt of this systematic review and meta-analysis was to evaluate the diagnostic accuracy of uNAG and provide comprehensive information for the accuracy of $\mathrm{UNAG}$, as a preventional biomarker in the early stages of DN in patients with T1DM and T2DM.

Anane et al. [33] in their research study showed that the values of uNAG and uNAG/Cr ratio from patients' urine samples, had an increasing rate as the values of albuminuria increased and the rate of glomerular infiltration (eGFR) decreased, in patients with T2DM compared to the control group. Sheira's et al. [41] study showed statistically significant increase of UNAG and UNAG/Cr ratio and decrease of the estimated GFR in patients with microalbuminuria compared to patients with normoalbuminuria and in all patient groups compared to control group. Moreover, a parallel increase of the urinary excretion of NAG with the deterioration of DN has been observed, which indicates the severity of kidney damage and disease progression [40,41]. Kim et al. in their study showed that the levels of urinary NAG had a moderate positive correlation with the levels of urinary ACR in T2DM and that increased levels in urinary NAG may be associated with glycemic parameters reflecting glucose fluctuation [53].

The main result of our meta-analysis is the high value of AUC for uNAG and uNAG/Cr in distinguishing the control group from normo-microalbuminuric diabetic patients. This finding shows that $\mathrm{uNAG/Cr}$ can be considered a potential, good biomarker to predict early diabetic nephropathy in patients with diabetes mellitus. Moreover, according to the guidelines by Swets, the diagnostic accuracy values of uNAG and uNAG/Cr showed moderate accuracy in the other settings as well. These findings support the hypothesis for renal tubule damage even in the initial stage stages of DN, before the presence of pathological amounts of albuminuria, indicating a promising diagnostic accuracy of the biomarker. It is worth mentioning that uNAG/Cr seemed to present higher capability than uNAG in the diagnosis of $\mathrm{DN}$, as designated by the comparison of the corresponding AUC values for each group.

According to the forest plot, there was significant presence of heterogeneity between studies. Possible causes of heterogeneity were mainly due to (a) the design of the studies which indicated different methodology of estimating creatine's concentration, (b) the different choice of urine collection for estimating ACR (spot urine or $24 \mathrm{~h}$ urine collection), and (c) the disparate race and ethnicity of the study population. In addition, a significant 
decrease in heterogeneity was observed among the category of diabetic patients with normoalbuminuria and diabetic patients with microalbuminuria. The low presence of heterogeneity was caused due to the common procedure followed in each study.

Furthermore, according to Deek's charts, publication bias was absent in the majority of studies. Publication bias was evident in studies that performed the discrimination between controls and normo-microalbuminuric diabetic patients using uNAG/Cr. This bias may be introduced due to unclear methodology used for estimating the values of microalbuminuria in some of the studies. As evidenced by the QUADAS quality assessment, the vague risk of bias in some studies on patient selection was present due to insufficient information about the diabetic patients' characteristics with normoalbuminuria and microalbuminuria.

In conclusion, the final results of this systematic review and meta-analysis indicate that UNAG is a promising biomarker (raw and creatinine-normalized) for early and valid prediction of diabetic nephropathy in patients with T1DM and T2DM. Meta-analysis findings indicate that uNAG/Cr has higher efficiency in all patient groups. In particular, higher accuracy was observed in identifying the presence of $\mathrm{DN}$ in normo-microalbuminuric patients with T1DM and T2DM.

Supplementary Materials: The following are available online at https:/ / www.mdpi.com/article/ $10.3390 /$ diabetology2040025/s1, Table S1. Clinical characteristics of the included studies in the meta-analysis for controls (healthy individuals) and diabetic patients with normoalbuminuria and microalbuminuria. Figures S1-S2. The hierarchical summary Receiver Operating Characteristic (hsROC) curve of uNAG and uNAG/Cr to discriminate normo-, micro-, normo/microalbuminuric diabetic patients. Figures S3-S5. Forest plot for sensitivity and specificity of uNAG and UNAG/Cr to distinguish normo-, micro-, normo/microalbuminuric diabetic patients. Figures S6-S8. Deek's funnel plot for the evaluation of publication bias of $\mathrm{UNAG}$ and $\mathrm{uNAG} / \mathrm{Cr}$.

Author Contributions: P.G.B. and G.V.K. conceived the study. A.R.D. and G.V.K. participated in data collection. A.R.D. performed the analysis. G.V.K. and P.G.B. participated in data collection and in the interpretation of the results. All authors participated in drafting the manuscript. All authors have read and agreed to the published version of the manuscript.

Funding: This research received no external funding.

Institutional Review Board Statement: Not applicable.

Informed Consent Statement: Not applicable.

Data Availability Statement: The data are given in the manuscript.

Conflicts of Interest: The authors declare no conflict of interest.

\section{References}

1. Nazar, C.M.J. Diabetic Nephropathy; Principles of Diagnosis and Treatment of Diabetic Kidney Disease. J. Nephropharmacol. 2014, 3, 15-20. [PubMed]

2. Deckert, T.; Feldt-Rasmussen, B.; Borch-Johnsen, K.; Jensen, T.; Kofoed-Enevoldsen, A. Albuminuria Reflects Widespread Vascular Damage. The Steno Hypothesis. Diabetologia 1989, 32, 219-226. [CrossRef] [PubMed]

3. KDOQI. Clinical Practice Guidelines and Clinical Practice Recommendations for Diabetes and Chronic Kidney Disease. Am. J. Kidney Dis. 2007, 49, S12-S154. [CrossRef]

4. Gross, J.L.; de Azevedo, M.J.; Silveiro, S.P.; Canani, L.H.; Caramori, M.L.; Zelmanovitz, T. Diabetic Nephropathy: Diagnosis, Prevention, and Treatment. Diabetes Care 2005, 28, 164-176. [CrossRef] [PubMed]

5. Mogensen, C.E. Microalbuminuria Predicts Clinical Proteinuria and Early Mortality in Maturity-Onset Diabetes. N. Engl. J. Med. 1984, 310, 356-360. [CrossRef]

6. Yaqoob, M.; Mcclelland, P.; Patrick, A.; Stevenson, A.; Mason, H.; White, M.; Bell, G. Evidence of Oxidant Injury and Tubular Damage in Early Diabetic Nephropathy. Mon. J. Assoc. Physicians 1994, 87, 601-607. [CrossRef]

7. Rossing, P.; Hougaard, P.; Parving, H.-H. Progression of Microalbuminuria in Type 1 Diabetes: Ten-Year Prospective Observational Study. Kidney Int. 2005, 68, 1446-1450. [CrossRef] [PubMed]

8. Hovind, P.; Tarnow, L.; Rossing, K.; Rossing, P.; Eising, S.; Larsen, N.; Binder, C.; Parving, H.-H. Decreasing Incidence of Severe Diabetic Microangiopathy in Type 1 Diabetes. Diabetes Care 2003, 26, 1258-1264. [CrossRef]

9. Bojestig, M.; Arnqvist, H.J.; Hermansson, G.; Karlberg, B.E.; Ludvigsson, J. Declining Incidence of Nephropathy in InsulinDependent Diabetes Mellitus. N. Engl. J. Med. 1994, 330, 15-18. [CrossRef] [PubMed] 
10. Caramori, M.L.; Fioretto, P.; Mauer, M. The Need for Early Predictors of Diabetic Nephropathy Risk: Is Albumin Excretion Rate Sufficient? Diabetes 2000, 49, 1399-1408. [CrossRef]

11. Jha, J.C.; Jandeleit-Dahm, K.A.M.; Cooper, M.E. New Insights into the Use of Biomarkers of Diabetic Nephropathy. Adv. Chronic Kidney Dis. 2014, 21, 318-326. [CrossRef]

12. von Eynatten, M.; Liu, D.; Hock, C.; Oikonomou, D.; Baumann, M.; Allolio, B.; Korosoglou, G.; Morcos, M.; Campean, V.; Amann, K.; et al. Urinary Adiponectin Excretion: A Novel Marker for Vascular Damage in Type 2 Diabetes. Diabetes 2009, 58, 2093-2099. [CrossRef]

13. Saraheimo, M.; Forsblom, C.; Thorn, L.; Wadén, J.; Rosengård-Bärlund, M.; Heikkilä, O.; Hietala, K.; Gordin, D.; Frystyk, J.; Flyvbjerg, A.; et al. Serum Adiponectin and Progression of Diabetic Nephropathy in Patients with Type 1 Diabetes. Diabetes Care 2008, 31, 1165-1169. [CrossRef] [PubMed]

14. Kanauchi, M.; Akai, Y.; Hashimoto, T. Transferrinuria in Type 2 Diabetic Patients with Early Nephropathy and Tubulointerstitial Injury. Eur. J. Intern. Med. 2002, 13, 190-193. [CrossRef]

15. Qin, L.-X.; Zeng, X.; Huang, G. Changes in serum and urine ceruloplasmin concentrations in type 2 diabetes. Zhong Nan Da Xue Xue Bao Yi Xue Ban 2004, 29, 208-211.

16. Vaidya, V.S.; Ferguson, M.A.; Bonventre, J.V. Biomarkers of Acute Kidney Injury. Annu. Rev. Pharmacol. Toxicol. 2008, 48, 463-493. [CrossRef] [PubMed]

17. Portilla, D.; Dent, C.; Sugaya, T.; Nagothu, K.K.; Kundi, I.; Moore, P.; Noiri, E.; Devarajan, P. Liver Fatty Acid-Binding Protein as a Biomarker of Acute Kidney Injury after Cardiac Surgery. Kidney Int. 2008, 73, 465-472. [CrossRef]

18. Nielsen, S.E.; Sugaya, T.; Tarnow, L.; Lajer, M.; Schjoedt, K.J.; Astrup, A.S.; Baba, T.; Parving, H.-H.; Rossing, P. Tubular and Glomerular Injury in Diabetes and the Impact of ACE Inhibition. Diabetes Care 2009, 32, 1684-1688. [CrossRef] [PubMed]

19. Kapoula, G.V.; Kontou, P.I.; Bagos, P.G. Diagnostic Accuracy of Neutrophil Gelatinase-Associated Lipocalin for Predicting Early Diabetic Nephropathy in Patients with Type 1 and Type 2 Diabetes Mellitus: A Systematic Review and Meta-Analysis. J. Appl. Lab. Med. 2019, 4, 78-94. [CrossRef]

20. Mungan, N.; Yuksel, B.; Bakman, M.; Topaloglu, A.K.; Ozer, G. Urinary N-Acetyl-beta-D-glucosaminidase Activity in Type I Diabetes Mellitus. Indian Pediatr. 2003, 40, 410-414. [PubMed]

21. Colhoun, H.M.; Marcovecchio, M.L. Biomarkers of Diabetic Kidney Disease. Diabetologia 2018, 61, 996-1011. [CrossRef]

22. Currie, G.; McKay, G.; Delles, C. Biomarkers in Diabetic Nephropathy: Present and Future. World J. Diabetes 2014, 5, 763-776. [CrossRef] [PubMed]

23. American Diabetes Association. Standards of Medical Care in Diabetes-2013. Diabetes Care 2013, 36, S11-S66. [CrossRef] [PubMed]

24. Whiting, P.F.; Rutjes, A.W.; Westwood, M.E.; Mallett, S.; Deeks, J.J.; Reitsma, J.B.; Leeflang, M.M.; Sterne, J.A.; Bossuyt, P.M.; QUADAS-2 Group. QUADAS-2: A revised tool for the quality assessment of diagnostic accuracy studies. Ann. Intern. Med. 2011, 155, 529-536. [CrossRef] [PubMed]

25. van Houwelingen, H.C.; Arends, L.R.; Stijnen, T. Advanced Methods in Meta-Analysis: Multivariate Approach and MetaRegression. Stat. Med. 2002, 21, 589-624. [CrossRef]

26. Arends, L.R.; Hamza, T.H.; van Houwelingen, J.C.; Heijenbrok-Kal, M.H.; Hunink, M.G.M.; Stijnen, T. Bivariate Random Effects Meta-Analysis of ROC Curves. Med. Decis. Mak. 2008, 28, 621-638. [CrossRef] [PubMed]

27. Higgins, J.P.; Green, S. Cochrane Handbook for Systematic Reviews of Interventions; Cochrane Book Series; John Wiley \& Sons: Hoboken, NJ, USA, 2019; p. 674.

28. Hozo, S.P.; Djulbegovic, B.; Hozo, I. Estimating the Mean and Variance from the Median, Range, and the Size of a Sample. BMC Med. Res. Methodol. 2005, 5, 13. [CrossRef] [PubMed]

29. Youden, W.J. Index for Rating Diagnostic Tests. Cancer 1950, 3, 32-35. [CrossRef]

30. Swets, J.A. Measuring the Accuracy of Diagnostic Systems. Science 1988, 240, 1285-1293. [CrossRef] [PubMed]

31. Higgins, J.P.T.; Thompson, S.G.; Deeks, J.J.; Altman, D.G. Measuring Inconsistency in Meta-Analyses. BMJ 2003, 327, 557-560. [CrossRef] [PubMed]

32. Deeks, J.J.; Macaskill, P.; Irwig, L. The Performance of Tests of Publication Bias and Other Sample Size Effects in Systematic Reviews of Diagnostic Test Accuracy Was Assessed. J. Clin. Epidemiol. 2005, 58, 882-893. [CrossRef]

33. Asare-Anane, H.; Twum, F.; Kwaku Ofori, E.; Torgbor, E.L.; Amanquah, S.D.; Osafo, C. Urinary Lysosomal Enzyme Activities and Albuminuria in Ghanaian Patients with Type 2 Diabetes Mellitus. Dis. Markers 2016, 2016, 2810639. [CrossRef]

34. Mohammadi-Karakani, A.; Asgharzadeh-Haghighi, S.; Ghazi-Khansari, M.; Hosseini, R. Determination of Urinary Enzymes as a Marker of Early Renal Damage in Diabetic Patients. J. Clin. Lab. Anal. 2007, 21, 413-417. [CrossRef] [PubMed]

35. Salem, M.A.K.; El-Habashy, S.A.; Saeid, O.M.; El-Tawil, M.M.K.; Tawfik, P.H. Urinary Excretion of N-Acetyl-Beta-DGlucosaminidase and Retinol Binding Protein as Alternative Indicators of Nephropathy in Patients with Type 1 Diabetes Mellitus. Pediatr. Diabetes 2002, 3, 37-41. [CrossRef]

36. Piwowar, A.; Knapik-Kordecka, M.; Fus, I.; Warwas, M. Urinary Activities of Cathepsin B, N-Acetyl-Beta-D-Glucosaminidase, and Albuminuria in Patients with Type 2 Diabetes Mellitus. Med. Sci. Monit. 2006, 12, CR210-CR214. [PubMed]

37. Ambade, V.; Sing, P.; Somani, B.L.; Basanna, D. Urinary N-Acetyl Beta Glucosaminidase and Gamma Glutamyl Transferase as Early Markers of Diabetic Nephropathy. Indian J. Clin. Biochem. 2006, 21, 142-148. [CrossRef] [PubMed] 
38. Assal, H.S.; Tawfeek, S.; Rasheed, E.A.; El-Lebedy, D.; Thabet, E.H. Serum Cystatin C and Tubular Urinary Enzymes as Biomarkers of Renal Dysfunction in Type 2 Diabetes Mellitus. Clin. Med. Insights Endocrinol. Diabetes 2013, 6, 7-13. [CrossRef] [PubMed]

39. Żurawska-Płaksej, E.; Ługowska, A.; Hetmańczyk, K.; Knapik-Kordecka, M.; Adamiec, R.; Piwowar, A. Proteins from the 18 Glycosyl Hydrolase Family Are Associated with Kidney Dysfunction in Patients with Diabetes Type 2. Biomarkers 2015, 20 , 52-57. [CrossRef]

40. Vaidya, V.S.; Niewczas, M.A.; Ficociello, L.H.; Johnson, A.C.; Collings, F.B.; Warram, J.H.; Krolewski, A.S.; Bonventre, J.V. Regression of Microalbuminuria in Type 1 Diabetes Is Associated with Lower Levels of Urinary Tubular Injury Biomarkers, Kidney Injury Molecule-1, and $N$-acetyl- $\beta$-D-glucosaminidase. Kidney Int. 2011, 79, 464-470. [CrossRef]

41. Sheira, G.; Noreldin, N.; Tamer, A.; Saad, M. Urinary Biomarker N-Acetyl- $\beta$-D-Glucosaminidase Can Predict Severity of Renal Damage in Diabetic Nephropathy. J. Diabetes Metab. Disord. 2015, 14, 4. [CrossRef]

42. Shimojo, N.; Kitahashi, S.; Naka, K.; Fujii, A.; Okuda, K.; Tanaka, S.; Fujii, S. Comparison of N-Acetyl-Beta-D-Glucosaminidase and Alanine Aminopeptidase Activities for Evaluation of Microangiopathy in Diabetes Mellitus. Metabolism 1987, 36, 277-280. [CrossRef]

43. Narita, T.; Hosoba, M.; Kakei, M.; Ito, S. Increased Urinary Excretions of Immunoglobulin g, Ceruloplasmin, and Transferrin Predict Development of Microalbuminuria in Patients with Type 2 Diabetes. Diabetes Care 2006, 29, 142-144. [CrossRef] [PubMed]

44. Navarro, J.F.; Mora, C.; Muros, M.; García, J. Urinary Tumour Necrosis Factor-Alpha Excretion Independently Correlates with Clinical Markers of Glomerular and Tubulointerstitial Injury in Type 2 Diabetic Patients. Nephrol. Dial. Transpl. 2006, 21, 3428-3434. [CrossRef] [PubMed]

45. Fujita, H.; Morii, T.; Koshimura, J.; Ishikawa, M.; Kato, M.; Miura, T.; Sasaki, H.; Narita, T.; Ito, S.; Kakei, M. Possible Relationship between Adiponectin and Renal Tubular Injury in Diabetic Nephropathy. Endocr. J. 2006, 53, 745-752. [CrossRef] [PubMed]

46. Kalansooriya, A.; Holbrook, I.; Jennings, P.; Whiting, P.H. Serum Cystatin C, Enzymuria, Tubular Proteinuria and Early Renal Insult in Type 2 Diabetes. Br. J. Biomed. Sci. 2007, 64, 121-123. [CrossRef] [PubMed]

47. Kalansooriya, A.; Jennings, P.; Haddad, F.; Holbrook, I.; Whiting, P.H. Urinary Enzyme Measurements as Early Indicators of Renal Insult in Type 2 Diabetes. Br. J. Biomed. Sci. 2007, 64, 153-156. [CrossRef]

48. 48 Fu, W.-J.; Xiong, S.-L.; Fang, Y.-G.; Wen, S.; Chen, M.-L.; Deng, R.-T.; Zheng, L.; Wang, S.-B.; Pen, L.-F.; Wang, Q. Urinary Tubular Biomarkers in Short-Term Type 2 Diabetes Mellitus Patients: A Cross-Sectional Study. Endocrine 2012, 41, 82-88. [CrossRef]

49. De Muro, P.; Lepedda, A.J.; Nieddu, G.; Idini, M.; Tram Nguyen, H.Q.; Lobina, O.; Fresu, P.; Formato, M. Evaluation of Early Markers of Nephropathy in Patients with Type 2 Diabetes Mellitus. Biochem. Res. Int. 2016, 2016, 7497614. [CrossRef] [PubMed]

50. Zhang, D.; Ye, S.; Pan, T. The Role of Serum and Urinary Biomarkers in the Diagnosis of Early Diabetic Nephropathy in Patients with Type 2 Diabetes. PeerJ 2019, 7, e7079. [CrossRef] [PubMed]

51. Nikolov, G.; Boncheva, M.; Gruev, T.; Biljali, S.; Stojceva-Taneva, O.; Masim-Spasovska, E. Urinary Biomarkers in the Early Diagnosis of Renal Damage in Diabetes Mellitus Patients. Scr. Sci. Med. 2013, 45, 58-64. [CrossRef]

52. Al-Hazmi, S.F.; Gad, H.G.M.; Alamoudi, A.A.; Eldakhakhny, B.M.; Binmahfooz, S.K.; Alhozali, A.M. Evaluation of Early Biomarkers of Renal Dysfunction in Diabetic Patients. Saudi Med. J. 2020, 41, 690-697. [CrossRef] [PubMed]

53. Kim, S.R.; Lee, Y.-H.; Lee, S.-G.; Kang, E.S.; Cha, B.-S.; Kim, J.-H.; Lee, B.-W. Urinary N-Acetyl- $\beta$-D-Glucosaminidase, an Early Marker of Diabetic Kidney Disease, Might Reflect Glucose Excursion in Patients with Type 2 Diabetes. Medicine 2016, 95 , e4114. [CrossRef] [PubMed] 